\title{
The Cellular Analog of Sensitization in Aplysia Emerges at the Same Time in Development as Behavioral Sensitization
}

\author{
Thomas G. Nolen ${ }^{1, a}$ and Thomas J. Carew ${ }^{2}$ \\ Departments of ${ }^{1,2}$ Psychology and ${ }^{2}$ Biology, Yale University, New Haven, Connecticut 06520, and the ${ }^{1}$ Marine Biological \\ Laboratory, Woods Hole, Massachusetts 02543
}

\begin{abstract}
Recent studies examining the development of learning and memory in the gill and siphon withdrawal reflex of Aplysia have shown that different forms of learning emerge according to very different developmental timetables. For example, in the previous paper, Rankin and Carew (1988) showed that, whereas habituation and dishabituation emerge early in Juvenile development (in stages 9 and 10, respectively), sensitization emerges at least $60 \mathrm{~d}$ later (in late stage 12). This developmental separation of different learning processes provides the opportunity to examine the unique contribution of specific cellular mechanisms to each form of learning. As a first step in this cellular analysis, in the present paper we have examined the development of the cellular analog of sensitization (facilitation of nondecremented EPSPs) in the identified giant neuron R2, which can serve as a monitor of the afferent input in the gill and siphon withdrawal reflex (Rayport and Camardo, 1984).

We have found 2 striking parallels between the development of behavioral sensitization and the development of its cellular analog: (1) Behavioral sensitization, produced by tail shock, emerges very late in juvenile development (stage 12), and the cellular analog of sensitization (produced by activation of the tail pathway) emerges by exactly the same late juvenile stage; (2) prior to the emergence of behavioral sensitization, tail shock unexpectedly was found to produce significant reflex depression (Rankin and Carew, 1988), and prior to the emergence of the cellular analog of sensitization, activation of the tail pathway was found to produce significant depression of the synaptic input in the reflex pathway. Thus, the cellular analog of sensitization in the CNS develops and matures in close temporal register with the development of behavioral sensitization in juvenile Aplysia.
\end{abstract}

The marine mollusc Aplysia has proven to be a very useful preparation for studying mechanisms of learning and memory on behavioral, cellular, and molecular levels (Kandel, 1976;

\footnotetext{
Received Mar. 3, 1987; revised June 15, 1987; accepted June 17, 1987.

We wish to thank our colleagues Emilie Marcus and Catharine Rankin for helpful comments throughout the project. We also are very grateful to the Howard Hughes Medical Institute, and to Tom Capo, for generously supplying juvenile Aplysia. This work was supported by NIH BRSG Grant 507-RR-075015 and NSF Grant BNS 8311300 (to T.J.C.) and by NIH NRSA 5-F32-NS-07480 and a fellowship from the Grass Foundation (to T.G.N.). Part of this project was carried out while T.G.N. was a Grass Summer Research Fellow at the Marine Biological Laboratory, Woods Hole. We thank Dr. Ronald R. Hoy for the generous loan of several pieces of equipment at the $\mathrm{MBL}$.

Correspondence should be addressed to Dr. Thomas J. Carew, Department of Psychology, P. O. Box 11A, Yale Station, New Haven, CT 06520.

aPresent address: University of Miami, Department of Biology, P.O. Box 249118, Coral Gables, FL 33124.

Copyright (C) 1988 Society for Neuroscience $0270-6474 / 88 / 010212-11 \$ 02.00 / 0$
}

Carew 1987; Hawkins et al., 1987). Recently, the utility of Aplysia has been extended by examining different forms of learning and memory as they emerge during development. For example, behavioral studies of the gill and siphon withdrawal reflex in juvenile Aplysia have shown that 3 forms of nonassociative learning - habituation, dishabituation, and sensitization-each emerge according to different developmental timetables: habituation emerges first, dishabituation emerges soon thereafter, and sensitization emerges at a much later developmental stage (Nolen et al., 1987; Rankin and Carew, 1987, 1988). Moreover, sensitization in another response system, escape locomotion, also emerges late in juvenile development, in close temporal register with sensitization in the gill and siphon withdrawal reflex (Stopfer and Carew, 1988).

The developmental separation of different learning processes in the gill and siphon withdrawal reflex of Aplysia affords the opportunity to examine the unique contribution of different cellular and molecular mechanisms to each form of learning. An important first step in this analysis is to establish that cellular analogs of these different forms of learning can be identified and analyzed in the CNS of juvenile Aplysia at the same stages of development that those forms of learning are first behaviorally expressed. The gill and siphon withdrawal reflex of juvenile Aplysia is mediated by central neural circuits located in the abdominal ganglion (Nolen et al., 1987; Rankin et al., 1987). In the abdominal ganglion, the giant mucus motor neuron R2 provides an excellent target neuron in which to explore cellular analogs of learning (Rayport and Camardo, 1984). This neuron is readily identifiable as a unique individual throughout juvenile development (Kriegstein, 1977a). In addition, $\mathrm{R} 2$ receives significant afferent input from the siphon (Kandel and Tauc, 1965; Rayport, 1981). Thus, R2 can serve as a cellular vantage point to monitor plastic changes that emerge in the reflex pathways for gill and siphon withdrawal.

The development of cellular analogs of habituation and dishabituation in R2 was first examined by Rayport (1981) and by Rayport and Camardo (1984). They found that the analog of habituation (synaptic decrement) was present at a very early stage in juvenile development, stage 9 , and that the analog of dishabituation (facilitation of decremented synaptic potentials) emerged in stage 10 , about a week later. Consistent with these cellular results were the behavioral observations of Rankin and Carew (1987), who found that habituation in the siphon withdrawal reflex was present in stage 9 and that dishabituation emerged in stage 10 . In the present paper we explore the developmental emergence of the cellular analog of sensitization (facilitation of nondecremented synaptic potentials) in $\mathrm{R} 2$. We found 2 striking parallels between the development of behavioral sensitization and the development of its cellular analog: 
First, behavioral sensitization (produced by tail shock) emerges very late in juvenile development-stage 12 (Rankin and Carew, 1988); the cellular analog of sensitization (produced by activation of the tail pathway) emerges in exactly the same late juvenile stage. Second, behavioral studies revealed that, prior to the emergence of sensitization, tail shock actually produced significant depression of the reflex responsc (Rankin and Carew, 1988). Our cellular analysis of sensitization revealed a parallel inhibitory process: prior to the emergence of the analog of sensitization, activation of the tail pathway produced significant depression of the afferent reflex input. Thus, there is a close temporal correspondence between the development and maturation of behavioral sensitization and a cellular reflection of that process in the CNS of juvenile Aplysia.

\section{Materials and Methods}

Animals. Laboratory-cultured postmetamorphic stage 12 juvenile Aplysia californica were obtained from the Howard Hughes Medical Institute mariculture facility, Woods Hole, Massachusetts, and maintained as described by Rankin and Carew $(1987,1988)$. Staging of juveniles followed the conventions of Kriegstein (1977b) and Rankin and Carew (1987). Stage 12 animals were further divided into 3 substages (Rankin and Carew, 1988) defined by wet weight and length: Early stage 12 animals ranged in weight from 0.05 to $0.14 \mathrm{gm}(9-12 \mathrm{~mm}$ in length); middle stage 12 animals weighed between 0.19 and $0.24 \mathrm{gm}$ (about $13-$ $15 \mathrm{~mm}$ in length); and late stage 12 animals weighed between 0.88 and $1.4 \mathrm{gm}(2-2.5 \mathrm{~cm}$ in length).

Physiological procedures. Larger juveniles (middle and late stage 12) were anesthetized by a hemocoel injection of approximately $0.5-1 \mathrm{ml}$ of a 1:1 dilution of isotonic $\mathrm{MgCl}_{2}(370 \mathrm{~mm})$ in Aplysia Ringer's [consisting of (in mM) $460 \mathrm{NaCl} ; 10.4 \mathrm{KCl} ; 11 \mathrm{CaCl}_{2} ; 55 \mathrm{MgCl}_{2}$, and 10 Tris $\mathrm{HCl}, \mathrm{pH}$ 7.8]. Smaller (early stage 12) animals were simply immersed in the $\mathrm{MgCl}_{2}$ /Ringer's solution. The anesthesia usually took effect within 5-10 min, after which the animal was pinned ventral side up to the bottom of a Sylgard-coated dish. An incision was made along the midline of the foot, and the abdominal ganglion was excised and placed in a small Sylgard-coated recording dish and washed in Ringer's for 15 min. The ganglion was stabilized for intracellular recording using micropins and glass suction electrodes (tip diameters, 20-75 $\mu \mathrm{m}$ I.D.) placed on the siphon nerve and on the right pleuro-abdominal conneclive.

Standard intracellular recording techniques were used (Castelluci et al., 1978; Carew et al., 1979). Recordings were made from the mucus motor neuron, R2, using micro-electrodes with resistances of $15-60$ $\Omega$. R2 can be readily identified early in development and can be recorded from as early as stage 9 (Kriegstein, 1977a; Rayport, 1981; Rayport and Camardo, 1984). The physiological preparation is shown in Figure 1 In late stage 12 animals, 2 independent microelectrodes were used, one to record membrane voltage and the other to pass current in order to hyperpolarize the neuron. Due to the smaller size of $R 2$ in early and middle stage 12 animals, a single recording microelectrode was used. An impalement was considered acceptable if the resting potential was $>40 \mathrm{mV}$ and the action potential $>60 \mathrm{mV}$. Voltage records were simultaneously displayed on a dual-beam oscilloscope and recorded on FM tape (Teac 1230 tape deck and Vetter model 2D FM recording adapter), as well as on a chart recorder (Grass polygraph or Gould 2400S).

Brief electrical pulses were delivered to the siphon nerve to evoke a complex EPSP in R2. Stimulus amplitude was standardized for all developmental stages as follows: After the cell was impaled, a single brief $(0.5-2 \mathrm{msec})$ shock was presented through the suction electrode to the siphon nerve once every 1-3 min, a nondecrementing interstimulus interval (ISI), at increasing amplitudes until an EPSP of 5-20 mV was evoked (this range of EPSP amplitude was typically achieved with a stimulus of $1-10 \mathrm{~V})$. To establish an effective voltage threshold for the facilitating stimulation to the connective (which carrics the input from the tail to the abdominal ganglion), a single brief shock (1-5 msec in duration) to the right connective was presented once every $2 \mathrm{~min}$ at increasing amplitudes until a 10-20 mV EPSP was evoked in R2. This synaptic input elicited 1 or 2 action potentials. In pilot studies we found that for heterosynaptic facilitation to be effective, the connective input to $\mathrm{R} 2 \mathrm{had}$ to be of sufficient magnitude to give rise to action potentials. Thus, in all experiments we increased the amplitude of connective stimulation above this threshold level. Once these threshold levels were established, stimulus parameters were not changed for the remainder of the experiment.

Two stimulus paradigms were used: (1) a sensitization analog, heterosynaptic facilitation of a nondecremented EPSP, and, in some cases, (2) a dishabituation analog, heterosynaptic facilitation of a homosynaptically depressed EPSP. Facilitation of the nondecremented EPSP involved presentation of 3 stimuli to the siphon nerve at a long, nondecrementing ISI of 1-3 min, followed by stimulation of the right connectivea $1.5 \mathrm{sec}$ train of shocks $(10 / \mathrm{sec})$ at the previously determined voltage threshold. Three more test stimuli were then delivered to the siphon nerve at the same ISI. In all cases, the ISI was maintained throughout the experiment; the time interval between connective stimulation and the next test stimulus was 5-20 sec. Facilitation of the decremented EPSP required, first, homosynaptic depression produced by presenting 15 stimuli to the siphon nerve at a decrementing ISI (5-10 sec) (Rayport and Camardo, 1984). Heterosynaptic facilitation was then produced by connective stimulation identical to that used to examine facilitation of the nondecremented EPSP. Finally, 3 more siphon nerve stimuli at the short ISI were presented to assess the degree of facilitation.

In examining facilitation of both decremented and nondecremented EPSPs following connective stimulation, the membrane potential was repolarized with extrinsic current (to the prestimulus level) to control for possible effects of anomalous rectification. In some experiments using a single electrode (in middle and early stage 12 animals), complete compensation was not possible due to bridge imbalance. However, in these cases, the depolarization from connective stimulation was relatively short-lived compared with instances of synaptic facilitation, which were still readily apparent after the membrane potential had returned to prestimulus levels.

Statistical analysis. Peak amplitude of the evoked EPSP was measured from chart records. In a few preparations, the facilitatory effects of connective stimulation were sufficient to cause previously subthreshold EPSPs to give rise to action potentials. In these cases, EPSP amplitude following connective stimulation was estimated as the largest subthreshold EPSP in the series. In assessing the degree of homosynaptic depression, each preparation contributed a single score, calculated as follows: $100 \times$ (average amplitude of the last 3 EPSPs of the decrement series)/ (the amplitude of the initial EPSP). In assessing heterosynaptic facilitation, cach prcparation contributcd a facilitation ratio, calculatcd as $100 \times$ [the average amplitude of the 3 postconnective stimulation (TEST) EPSPs]/(the average of the 3 PRE-connective stimulation EPSPs). Thus, depression and facilitation scores were computed as a percentage of (1) the initial response or (2) of the average PRE-connective stimulation response, respectively.

As the data were non-normally distributed, nonparametric statistics were used for the analyses (Siegel, 1956). Between- and within-group comparisons were made using Mann-Whitney $U$ tests for independent samples, and Wilcoxon signed-rank tests for correlated samples, respectively (Siegel, 1956). Unless otherwise indicated, data are expressed as medians \pm interquartile ranges (IQR). Significant occurrence of facilitation within and between the various substages was assessed using sign tests and the $G$-test for a $3 \times 2$ contingency table (Siegel, 1956; Sokal and Rohlf, 1969), respectivcly. For these comparisons, a previously subthreshold (TEST) EPSP was scored as facilitated if, following connective stimulation, it either produced an action potential or was larger than its peak amplitude prior to connective stimulation. All significance levels are 2-tailed.

\section{Results}

\section{Late stage 12}

\section{Cellular analogs of dishabituation and sensitization}

Using the preparation shown in Figure 1, we first examined the cellular analogs of dishabituation and sensitization in late stage 12 animals. The analogs of dishabituation and sensitization were studied by determining the effects of connective stimulation on decremented and nondecremented complex EPSPs, respectively, in the identified mucus motor neuron R2 (see Materials and Methods). Examples of our results are shown in Figure 2. Connective stimulation produced pronounced heterosynaptic facil- 
Figure 1. Physiological preparation for the examination of the development of the cellular analogs of dishabituation and sensitization. Intracellular electrodes were used to record the membrane potential $\left(V_{m}\right)$ of the identified mucus motorneuron R2 in the abdominal ganglion of stage 12 animals. In late stage 12 animals, a second electrode $\left(I_{\text {inj }}\right)$ was used to hyperpolarize the cell. In early and mid-stage 12 animals, hyperpolarizing current was passed through the recording electrode by means of a bridge circuit. Afferent input from the siphon was activated by a brief shock to the siphon nerve, which evoked a complex EPSP in R2. Input from the tail pathway was activated by a train of brief shocks to the right connective.

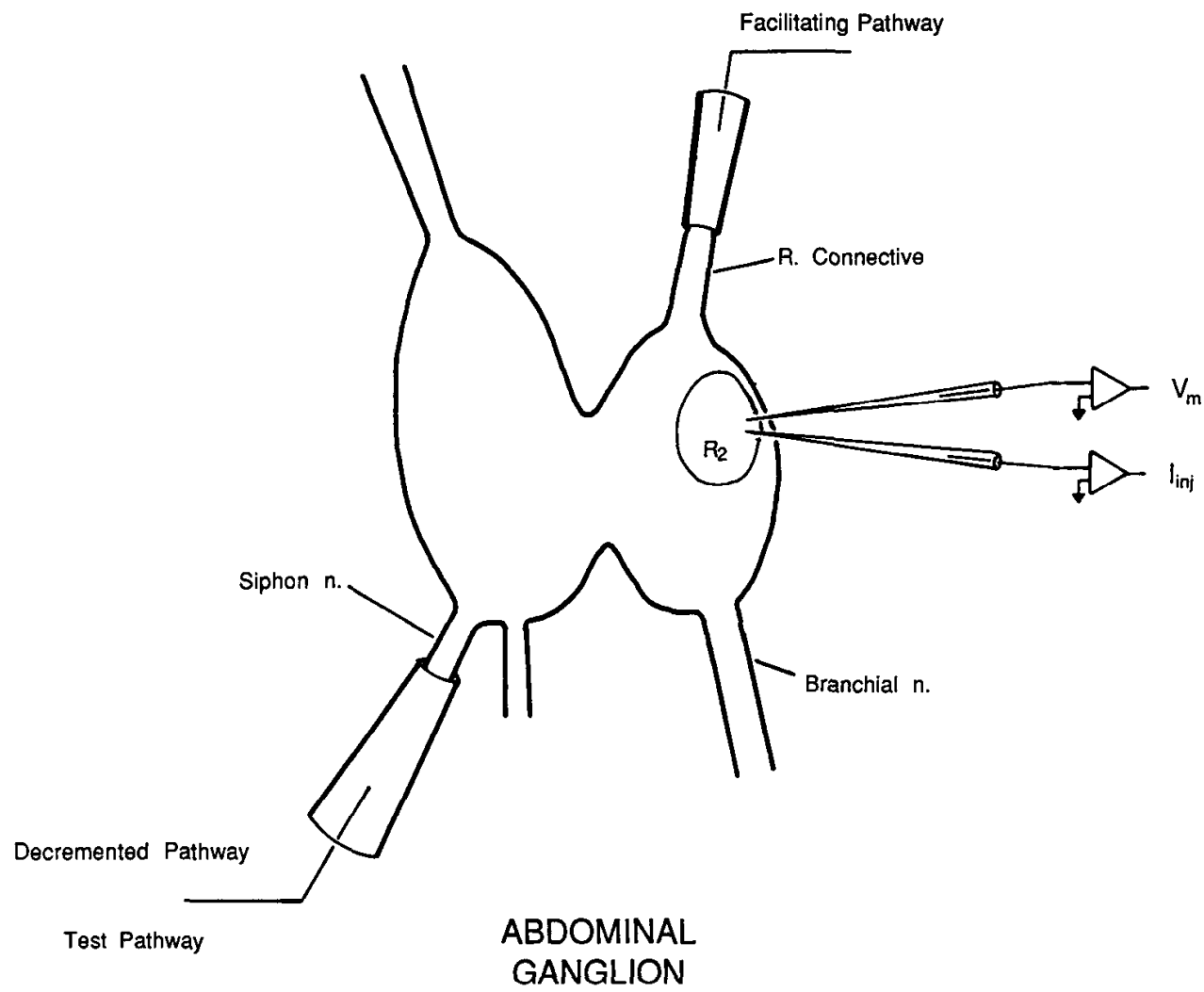

itation of both decremented and nondecremented EPSPs. In both cases, facilitation was usually apparent within 5-10 sec and generally lasted for at least $6 \mathrm{~min}$ (as long as was observed).

In all, 13 experiments were carried out. Facilitation of both decremented and nondecremented EPSPs was examined in each preparation. Thus, each experiment consisted of 2 phases, with a minimum of $10 \mathrm{~min}$ rest between them. The experiments were counterbalanced: in half, decremented EPSPs were examined first; in the other half, nondecremented EPSPs were examined first. In 8 experiments, the magnitude and time course of facilitation could be accurately measured throughout the entire duration of both phases of the experiments. These results are shown in Figure 3. Figure $3 A$ shows results in which the EPSP was homosynaptically depressed by repeated siphon nerve stimulation at a short ISI $(10 \mathrm{sec})$. The amplitude of the EPSP decremented to $55 \%$ of the control value by the 15 th stimulus. Both the time course and level of decrement we observed were comparable to that reported by Rayport and Camardo (1984) in stage 9 juveniles and in adult R2 at the same ISI (unpublished observations). Connective stimulation invariably facilitated the decremented EPSP, usually to about the level of the initial EPSP, and sometimes higher. Figure $3 B$ shows heterosynaptic facilitation of the nondecremented EPSP. A longer ISI ( $3 \mathrm{~min}$ ) produced a stable, nondecremented baseline. Within 5-20 sec of connective stimulation, the evoked EPSP increased to a mean of $143 \%$ of its initial amplitude. The EPSP amplitude subsequently decreased to $116 \%$ within 6 min (Fig. $3 B$, Stim 6) and usually returned to the initial baseline within $15 \mathrm{~min}$.

A statistical analysis revealed that a significant number of preparations exhibited facilitation of both decremented EPSPs $(13 / 13, p<0.006)$ and nondecremented EPSPs $(12 / 13, p<$ $0.006)$. Within-group comparisons wcre next carricd out to de- termine whether there was significant facilitation of decremented and nondecremented EPSPs compared with their own respective baselines.

Since there were 2 phases in each experiment, we first determined whether there was a significant order effect, that is, whether facilitation in the second phase was different than that in the first phase. We did observe a modest effect: Independent of whether decremented or nondecremented EPSPs were examined in the second phase, facilitation in the second phase was on average weaker (by about 15-25\%) than in the first phase. However, this difference was not statistically significant. Thus, for each stimulus paradigm, the first and second phase data were pooled for a within-group analysis. A summary $(n=13)$ is presented in Figure 4 which shows the change in EPSP amplitude following connective stimulation for both decremented and nondecremented EPSPs. In decremented trials, prior to connective stimulation, significant homosynaptic depression was produced by the 15 stimuli with a $10 \mathrm{sec}$ ISI (median, 62\% relative to the initial EPSP, $p<0.001$ ). Moreover, significant facilitation was evident for both groups: DECREMENTED, median $=149 \%, p<0.001$; NONDECREMENTED, median $=$ $126 \%, p<0.001$. Thus, extending previous results of Rayport and Camardo (1984), our data show that the cellular analog of dishabituation, facilitation of decremented EPSPs, is present in late stage 12. Moreover, our data also show that the cellular analog of sensitization, facilitation of nondecremented EPSPs, is also present in late stage 12 of juvenile development.

The primary aim of this paper was to examine the developmental timetable for the emergence of the cellular analog of sensitization. Thus, in the earlier developmental stages (middle stage 12 and early stage 12), we focused our attention on the facilitation of nondccremented EPSPs. 


\section{LATE STAGE 12}

A

Decremented<smiles>CCCCCCCC</smiles>

STIM 1

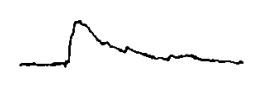

14

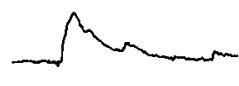

15

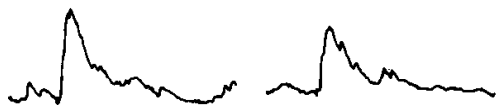

16

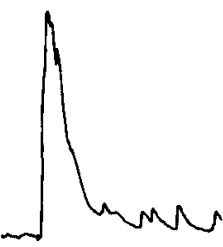

4

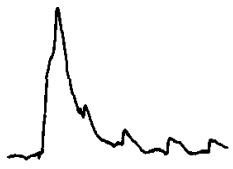

5

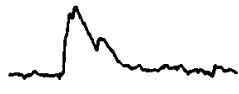

18

B Non-Decremented

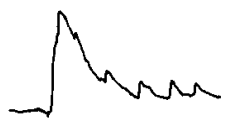

STIM 1

2

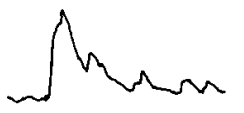

3

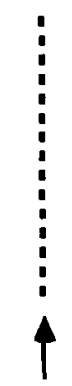

r. con. stim
$13 \mathrm{mV}$

Figure 2. Analogs of dishabituation and sensitization in R2 of a late stage 12 animal. $A$, Analog of dishabituation (heterosynaptic facilitation of a decremented EPSP): Repeated stimulation of the siphon nerve at a $10 \mathrm{sec}$ ISI produced significant decrement of the evoked EPSP. The EPSPs in response to stimuli 1,14 , and 15 (prior to connective stimulation) and stimuli 16-18 (following connective stimulation) are shown. Connective stimulation (arrow and dashed line) resulted in heterosynaptic facilitation of the EPSP, which redecremented with continued stimulation at the 10 sec ISI. $B$, Analog of sensitization (heterosynaptic facilitation of a nondecremented EPSP): Stimulation of the siphon nerve at a 3 min ISI produced no significant decrement. Connective stimulation produced strong facilitation of the evoked EPSP which decayed over the 6 min test period. A 10 min rest period was interposed between the experiments shown in $A$ and $B$.

A

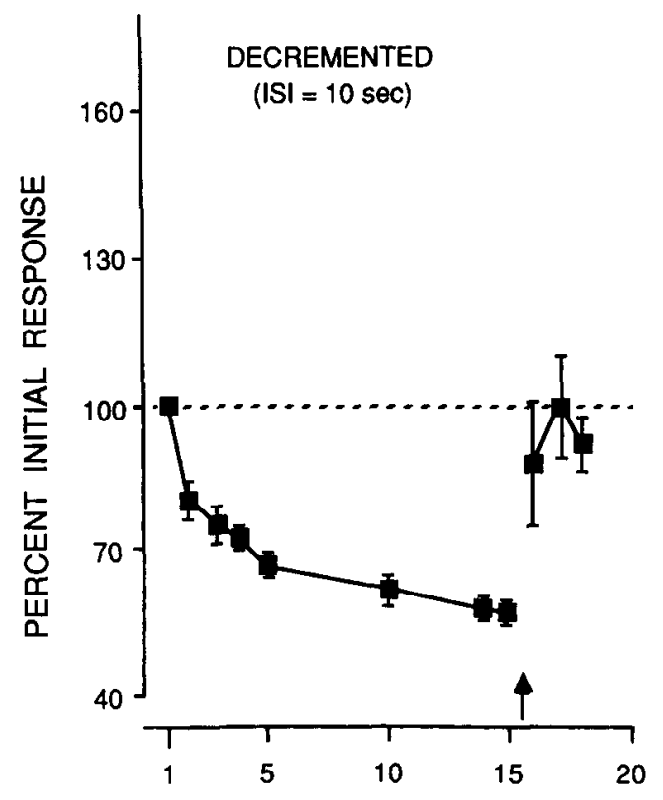

B

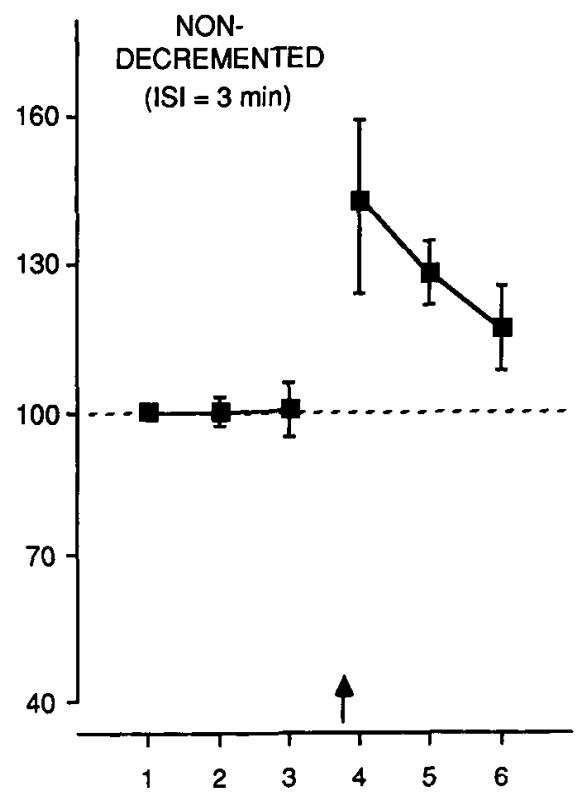

Figure 3. Summary of the magnitude and time course of the analog of dishabituation $(A)$ and sensitization $(B)$. The data, plotted as a percentage of the initial EPSP amplitude, are the means ( \pm SEM) from 8 late stage 12 animals in which the facilitation of both decremented and nondecremented EPSPs was examined: In half of the preparations, decremented EPSPs were examined first, while in the other half, nondecremented EPSPs were examined first. The facilitating stimulus (right connective stimulation) is indicated at the arrow. There was a minimum rest period of $10 \mathrm{~min}$ between the 2 experiments. 
LATE STAGE 12

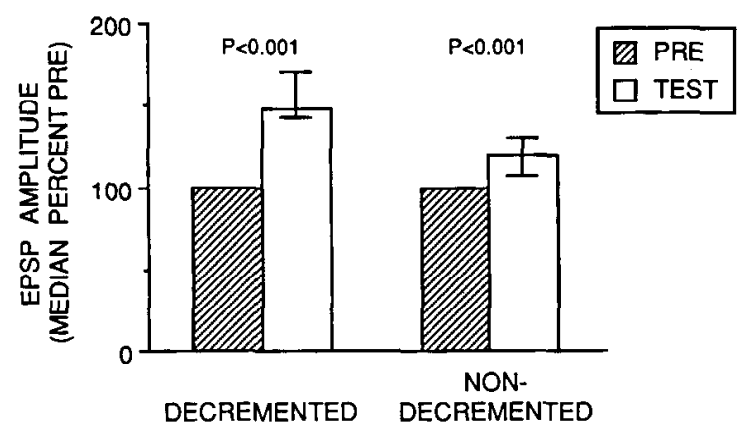

Figure 4. Comparison of the facilitation of decremented and nondecremented EPSPs in late stage 12. The medium EPSP amplitude $(n=13)$ \pm Interquartile range (IQR), are expressed as a percentage change (facilitation ratio, see text) following connective stimulation (TEST). Significant facilitation was observed for both decremented and nondecremented EPSPs $(p<0.001$ in each case).

\section{Middle stage 12}

The same experimental procedures were used to examine facilitation of the complex EPSP as in the previous experiments, with the exception that a $1 \mathrm{~min}$ (nondecrementing) ISI was used (see Materials and Methods). An example of our results is shown in Figure 5. As in late stage 12, connective stimulation produced clear facilitation of nondecremented EPSPs in middle stage 12. Figure 5 shows an example in which it was possible to quantify the magnitude of facilitation by expressing the amplitude of the complex EPSP after connective stimulation as a percentage of the preshock level (in this example, 217\%).

A summary of the experiments in middle stage $12(n=8)$ is shown in Figure 6. Connective stimulation produced significant facilitation of the nondecremented EPSP at this stage: The magnitude of facilitation was $135 \%$. As in late stage 12 , the onset of facilitation was rapid. However, the duration of facilitation in middle stage 12 appeared to be somewhat shorter than in late stage 12 (compare Figs. 2 and 5). In summary, the analog of sensitization is present in middle stage 12 of juvenile development.

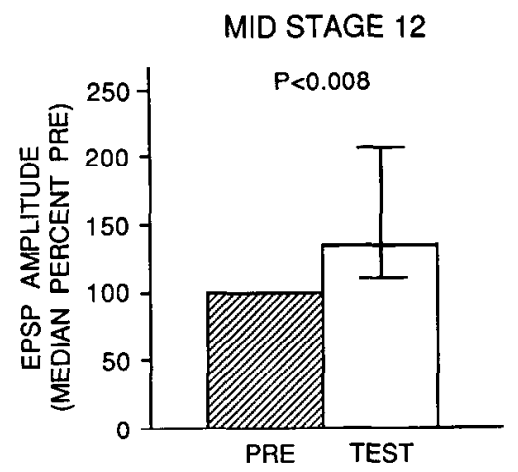

Figure 6. Summary of middle stage 12 experiments. Medium EPSP amplitude $( \pm I Q R)$ for the average of TEST trials $1-3$, relative to the PRE-test period $(n=8)$, is shown. Significant facilitation was evident in the test period following connective stimulation.

\section{Early stage 12}

Cellular analog of sensitization is absent in early stage 12

Having established that the analog of sensitization was present in both late and middle stage 12 , we next turned our attention to early stage 12 . In contrast to the previous substages studied, facilitation of nondecremented EPSPs was absent in this substage. A typical result is shown in Figure 7. Following connective stimulation, rather than exhibiting facilitation, the EPSP actually exhibited marked depression (see below). This lack of facilitation in early stage 12 was observed in 7 of 8 preparations. In one, modest facilitation was observed.

A summary of the experiments in early stage $12(n=8)$ is shown in Figure 8. Connective stimulation produced no significant facilitation of nondecremented EPSPs in this developmental substage when averaged over the 3 TEST trials (not shown: median facilitation $=54 \%, p>0.11$ ). Rather, it produced marked synaptic depression. In fact, as shown in Figure 8 , significant synaptic depression was evident in the first TEST EPSP following connective stimulation ( $p<0.03$, see below).

The failure to observe facilitation in early stage 12 was not the result of ineffective activation of the input from the right connective. As described in Materials and Methods, prior to the cxperiment we were careful to ensure that connective stimulation produced strong synaptic input to $\mathrm{R} 2$ that gave rise to action

\section{MID STAGE 12}

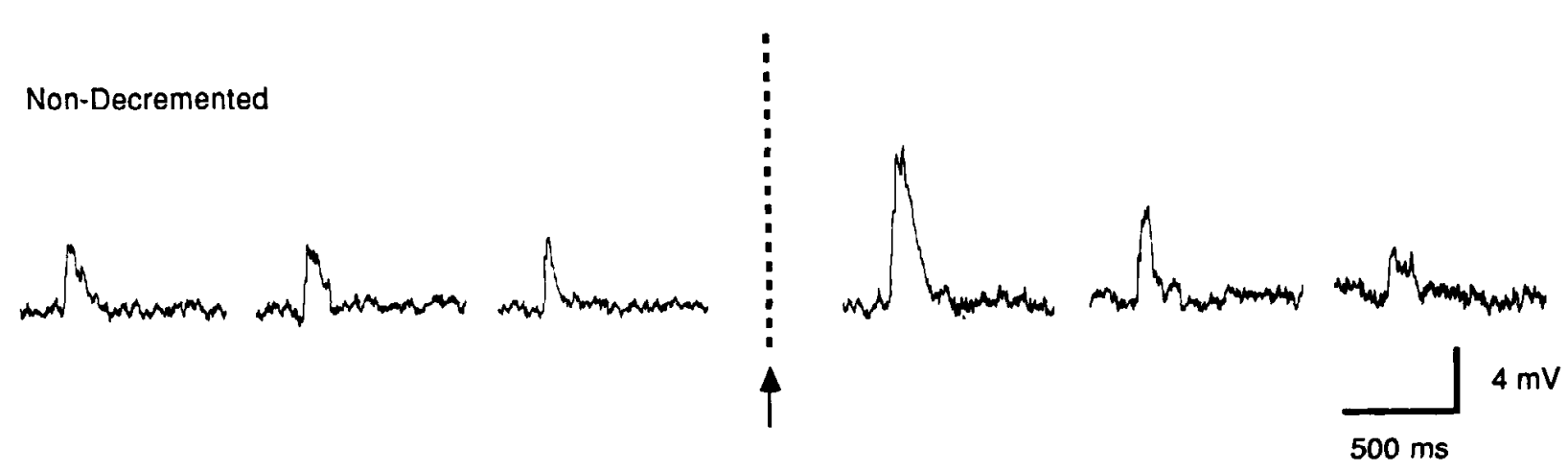

Figure 5. Analog of sensitization in middle stage 12. Stimulation of the siphon nerve at a 1 min ISI produced a stable baseline EPSP, which was markedly facilitated following connective stimulation. 


\section{EARLY STAGE 12}

Non-Decremented

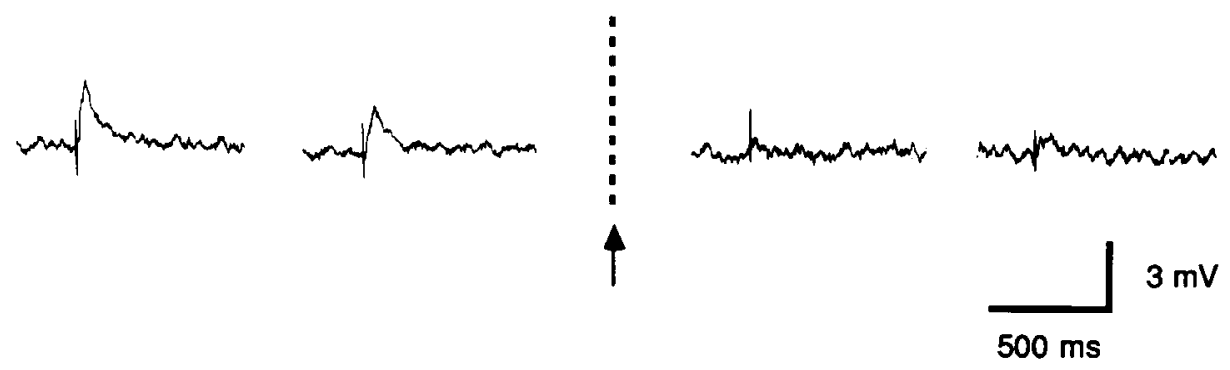

Figure 7. Analog of sensitization is lacking in early stage 12 . In contrast to the older developmental stages (e.g., see Figs. 2 and 5), rather than producing facilitation, connective stimulation actually caused marked inhibition of the EPSP (ISI = $1 \mathrm{~min})$.

potentials. In addition, we found that identical connective stimulation produced significant facilitation of nondecremented EPSPs in middle and late stage 12 (Figs. 4, 6). Finally, in early stage 12 , connective stimulation could produce clear facilitation of decremented, EPSPs (the analog of dishabituation) in the same preparation in which facilitation of nondecremented EPSPs was absent (an example is shown in Figs. 9 and 10). This result confirms an earlier report by Rayport and Camardo (1984), who found that connective stimulation produced clear facilitation of decremented EPSPs in R2 in this and much earlier developmental stages. Thus, our results support the conclusion that, in parallel to the behavioral observation that sensitization is absent in early stage 12 (Rankin and Carew, 1988), the cellular mechanisms underlying facilitation of nondecremented EPSPs are also absent in this early substage.

A summary of the results from all developmental stages that we examined is shown in Figure 11. A clear developmental trend in the emergence of the analog of sensitization is apparent. Whereas significant facilitation of nondecremented EPSPs is evident in late and middle stage $12(p<0.001$ and $p<0.008$, respectively), no significant occurrence of facilitation is evident in early stage 12 . Moreover, significantly more middle and late stage 12 preparations showed facilitation of nondecremented EPSPs than did early stage 12 preparations $(p<0.01)$. Thus, our data show that the cellular analog of sensitization emerges sometime between early and middle stage 12 of juvenile development.

\section{Analysis of nondecremented EPSPS reveals an inhibitory effect of connective stimulation}

Since the emergence of facilitation seems to occur between early and middle stage 12 , we carried out a detailed comparison of the effects of connective stimulation in these 2 developmental substages. Such a comparison is illustrated in Figure 12, in which the EPSP amplitude in each of the tests following connective stimulation is expressed as a percentage of its amplitude before connective stimulation [i.e., $100 \times$ (TEST EPSP amplitude)/ (average PRE EPSP amplitude)]. A striking difference emerges between the 2 substages: Net facilitation is observed in middle stage 12, whereas net depression is observed in early stage 12 . A within-group analysis of the middle stage 12 preparations revealed significant facilitation in TESTs 1 and $2(p<0.008$ and $p<0.016$, respectively) produced by connective stimulation. In contrast, a within-group analysis of the early stage 12 preparations revealed that connective stimulation produced a significant depression of the EPSP in R2 (TEST $1, p<0.03$ ).
As was the case for facilitation in middle stage 12 , the time course of this synaptic depression in early stage 12 could last several minutes. In addition, a between-group analysis revealed a clear and significant difference between the 2 substages. In early stage 12, the relative amplitudes of EPSPs in TESTs 1 and 2 were significantly less than those of middle stage $12(p<0.007$ and $p<0.01$, respectively). The difference between the effects of connective stimulation in these 2 substages can be best appreciated by examining the first TEST EPSP following connective stimulation, as shown in Figure 13. Whereas all middle stage 12 preparations exhibited facilitation, all but one early stage 12 preparation exhibited depression; and there was very little overlap between the groups $(p<0.007)$.

The reduction in EPSP amplitude could result partly from the shunting effects of connective stimulation. However, 2 observations suggest the hypothesis that shunting alone may not account for all of the synaptic depression observed. First, the depression can be quite long lasting (several minutes-see Figs. 7 and 12). The shunting effects of connective stimulation are not likely to last more than $30-60 \mathrm{sec}$, estimated by the time course of depolarization produced by connective stimulation. Second, connective stimulation in early stage 12 can completely depress the synaptic potential (see Fig. 7). The magnitude of this inhibitory effect suggests that shunting alone is unlikely to account for all the synaptic depression we observed. However, direct measurement of input conductance during the synaptic depression will be necessary to assess accurately the degree to which shunting accounts for the inhibitory effects of connective

\section{EARLY STAGE 12}

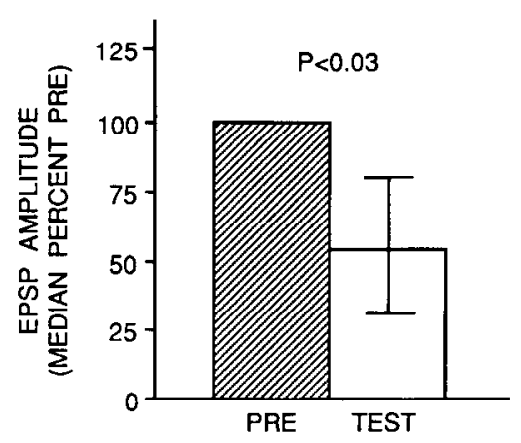

Figure 8. Summary of early stage 12 experiments. The median EPSP $( \pm \mathrm{IQR})$ relative to the PRE-test baseline is shown for TEST trial 1. Significant depression $(p<0.03)$ was evident within $20-30 \mathrm{sec}$ of connective stimulation. (ISI $=1 \mathrm{~min}$ ). 


\section{EARLY STAGE 12}

Figure 9. Connective stimulation facilitates decremented but not nondecremented EPSPs in early stage 12. $A$, EPSPs were evoked by siphon nerve stimulation at a nondecrementing ISI of $1 \mathrm{~min}$. Connective stimulation ( $\mathrm{ar}$ row) did not facilitate the evoked EPSP. $B$. At $10 \mathrm{~min}$ after the experiment in $A$, the EPSP was decremented by siphon nerve stimulation at a $5 \mathrm{sec}$ ISI; subsequent identical connective stimulation as in part $\boldsymbol{A}$ strongly facilitated the decremented EPSP.

\section{A \\ NON-DECREMENTED}<smiles>C1CCCCCC1</smiles>

STIM

B

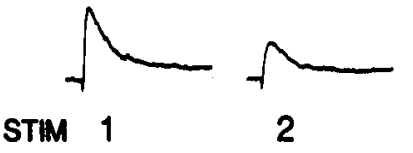

3

2

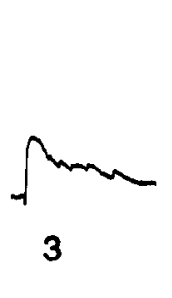

个
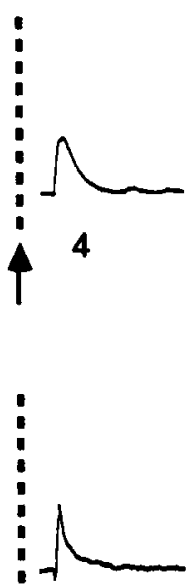

15

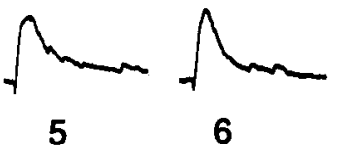

5

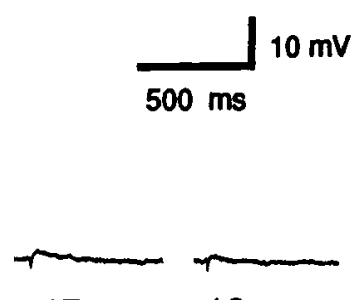

17 stimulation in this early developmental substage. In summary, our results show that an inhibitory component of connective stimulation is revealed when nondecrementing EPSPs are examined in early stage 12 , prior to the emergence of the analog of sensitization.

\section{Discussion}

\section{Cellular analogs of habituation, dishabituation, and} sensitization have different developmental timetables in Aplysia

In previous behavioral studies of the gill and siphon withdrawal reflex of juvenile Aplysia, Rankin and Carew $(1987,1988)$ found that the 3 principal forms of nonassociative learning-habituation, dishabituation, and sensitization-emerged according to different developmental timetables. Habituation was present in stage 9, dishabituation emerged about a week later in stage 10 , and sensitization emerged several weeks after dishabituation, in late stage 12 . In order to study the cellular events underlying the expression of these different forms of learning, it is important to show that cellular analogs for each of them can be identified in the CNS of juvenile Aplysia and that these analogs exhibit a developmental time course parallel to the behavioral expression of the learning.

The identified mucus motor neuron $\mathrm{R} 2$ provides an excellent system for examining the developmental emergence of cellular analogs of learning in the siphon and gill withdrawal reflex for 3 reasons: (1) $\mathrm{R} 2$ can be identified as a unique individual throughout juvenile development (Kriegstein, 1977a); (2) the neuron receives afferent input from the siphon reflex pathway (Kandel and Tauc, 1965; Rayport, 1981); and (3) the neuron receives facilitatory input from the tail via the pleuro-abdominal connectives (Kandel and Tauc, 1965; Rayport, 1981). The detailed circuitry for the siphon afferent input to $\mathrm{R} 2$, compared to the gill and siphon motor neurons, is not yet known. However, both mono- and polysynaptic afferent connections to R2 and to the gill and siphon motor neurons show very comparable synaptic plasticity (Rayport, 1981; and unpublished observations). Thus, $\mathrm{R} 2$ can serve as a reliable cellular monitor of plasticity in the afferent input to the gill and siphon withdrawal reflex.

The developmental emergence of the cellular analogs of habituation (synaptic decrement) and dishabituation (facilitation of decremented synaptic potentials) in $\mathrm{R} 2$ was first examined
Figure 10. Summary of physiological data shown in Figure 9. $A$, Non-decremented EPSPs was not facilitated by connective stimulation. $B$, However, in the same preparation $10 \mathrm{~min}$ later, identical connective stimulation produced clear facilitation of a decremented EPSP.
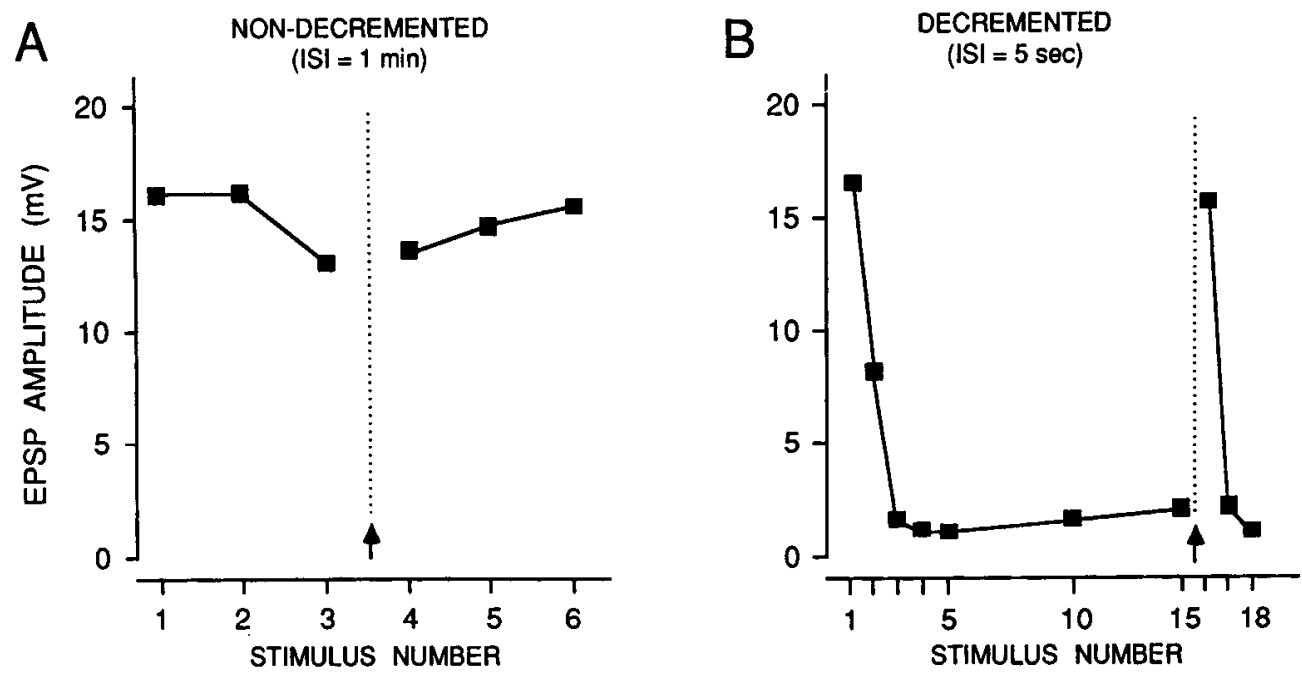


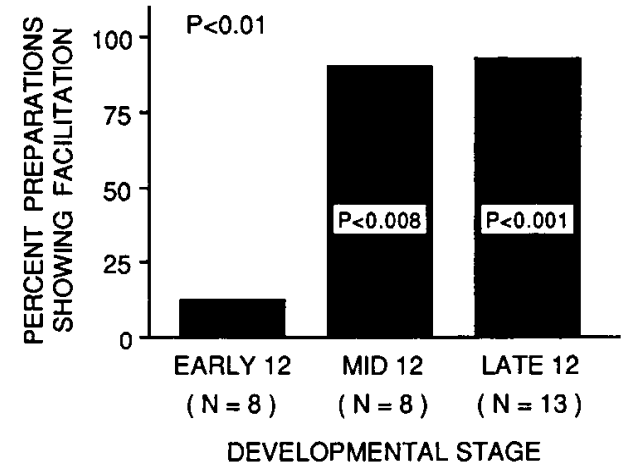

Figure 11. Development of the cellular analog of sensitization. The percentage of preparations exhibiting facilitation of the evoked EPSP following connective stimulation is shown for each developmental substage examined. Significant facilitation is evident in late and middle stage 12 but absent in early stage 12 . There is a significant trend $(p<$ 0.01 ) in the emergence of facilitation over these 3 substages of development.

by Rayport (1981) and Rayport and Camardo (1984). They found that homosynaptic depression could be observed in R2 as early as stage 9 and that facilitation of depressed synaptic potentials emerged at a distinct and later stage, stage 10. In the present study, we examined the emergence of the cellular analog of sensitization (facilitation of nondecremented synaptic potentials) in R2. In contrast to the emergence of the analog of dishabituation, we found that the analog of sensitization in $\mathrm{R} 2$ did not emerge until many weeks later, between early and middle stage 12. Morcover, confirming and extending Rayport and $\mathrm{Ca}$ mardo (1984), we found the cellular analog of dishabituation was present throughout stage 12 (including the earliest substage, where the analog of sensitization was absent). Finally, the cellular analogs of habituation, dishabituation, and sensitization in R2 (Kandel and Tauc, 1965; Rayport and Camardo, 1984; and the present work) were quite comparable to those reported in adult Aplysia in motorneurons mediating the gill and siphon withdrawal reflex (Castellucci et al., 1970; Kupfermann et al., 1970; Carew et al., 1971).

A comparison of the developmental timetables for behavioral habituation, dishabituation, and sensitization and their cellular analogs is given in Figure 14. A clear parallel for each form of learning is apparent: Habituation and its analog, synaptic decrement, emerge by stage 9 ; dishabituation and its analog, facilitation of decremented EPSPs, emerge in stage 10; and sensitization and its analog, facilitation of nondecremented EPSPs, emerge considerably later, in stage 12 . The time resolution of these studies, utilizing discrete, nonoverlapping developmental stages, permits us to ascertain that the behavioral expression of the learning and their cellular analogs emerge in close temporal register. However, further studies with finer-grained temporal resolution will be required to specify the precise time of emergence of these processes. Moreover, the gill and siphon motor neurons have not yet been identified at early stages of juvenile development. It will obviously be important to examine the emergence of different analogs of learning in these circuit elements as they are identified. Nonetheless, the close developmental parallel between the emergence of each form of learning and its respective cellular analog in $\mathrm{R} 2$ is striking and suggests that gaining an understanding of the cellular mechanisms underlying the emergence of the synaptic plasticity in the cellular analogs may contribute importantly to an understanding of the mechanisms underlying the learning processes themselves.

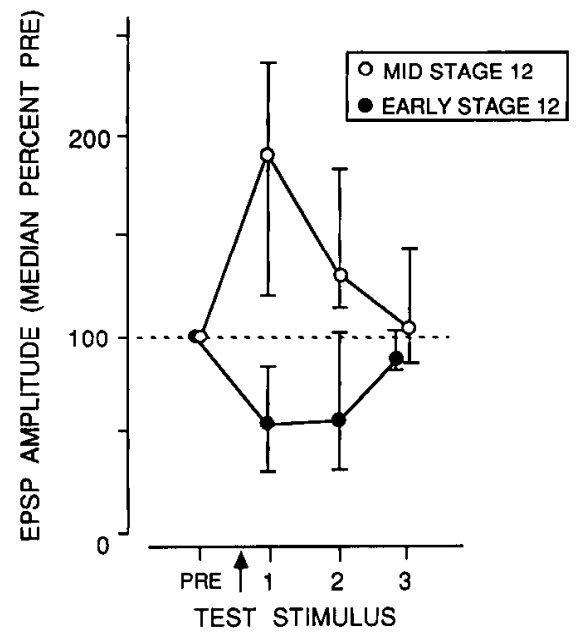

Figure 12. Comparison of the effects of connective stimulation on nondecremented EPSPs in middle and early stage 12. Median EPSP amplitude $( \pm I Q R)$ for each test stimulus following connective stimulation (arrow) is plotted as a percentage of the average PRE-test amplitude. The ISI was $1 \mathrm{~min}$ in each experiment. Connective stimulation produced clear facilitation in middle stage 12 but depression in early stage 12: Significant facilitation was observed in middle stage 12 at TESTs 1 and $2(p<0.008$ and $p<0.016)$ and significant inhibition in early stage 12 at TEST $1(p<0.03)$. Moreover, early and middle stage 12 preparations were significantly different from each other at TESTs 1 and $2(p<0.007$ and $p<0.01$, respectively).

\section{Analysis of nondecremented EPSPs prior to the emergence of sensitization reveals an inhibitory process}

In the behavioral analysis described in the preceding paper, Rankin and Carew (1988) found that, prior to the emergence of sensitization in late stage 12 , tail shock actually produced an unexpected and significant depression of nondecremented reflex responses. An interesting cellular analog of this depressive effect

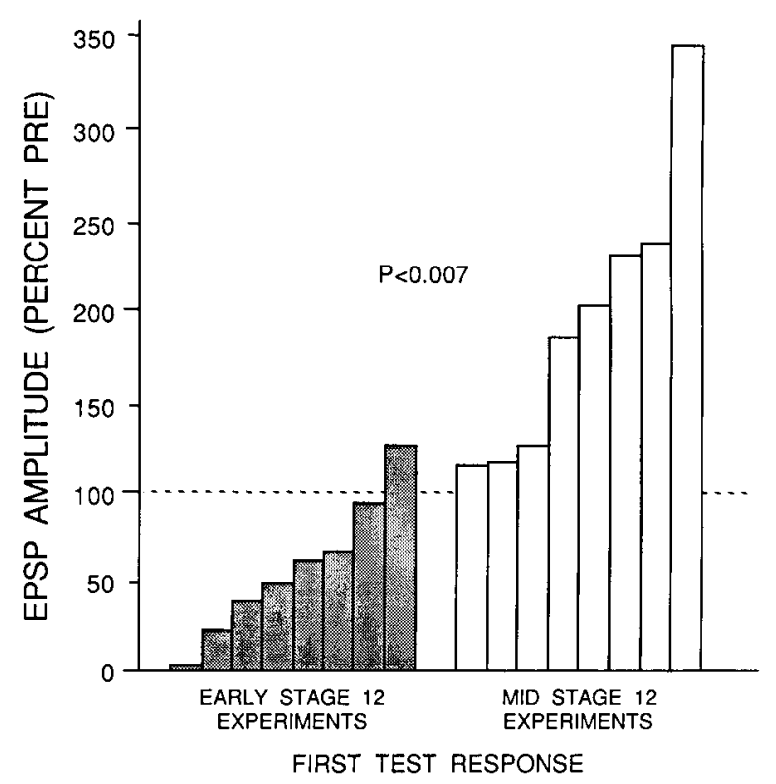

Figure 13. Comparison of the effects of connective stimulation in early and middle stage 12 in TEST 1 . The amount of facilitation of the first test response following connective stimulation is plotted for each preparation. All middle stage 12 preparations (white bars) exhibited facilitation, whereas all but one early stage 12 preparation showed depression (shaded bars). There was a highly significant difference between the 2 substages $(p<0.007)$. 


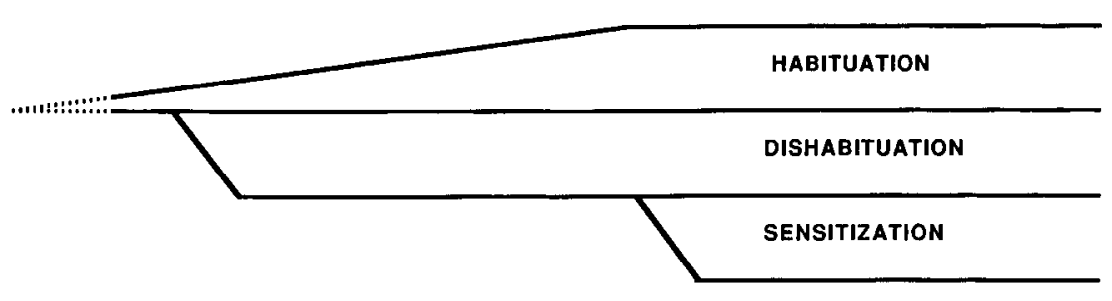

Figure 14. Comparison of the developmental timetables of behavioral habituation, dishabituation, and sensitization and thcir cellular analogs. Several forms of nonassociative learning emerge in the gill and siphon withdrawal reflex at different times during juvenile development. Habituation and its cellular analog (homosynaptic decrement of EPSPs in R2) emerge by stage 9; dishabituation and its cellular analog (facilitation of decremented EPSPs in R2) emerge in stage 10 . Sensitization and its cellular analog (facilitation of nondecremented EPSPs in R2) emerge much later (approximately $60 \mathrm{~d}$ ) in middle to late stage 12 (see text for details).

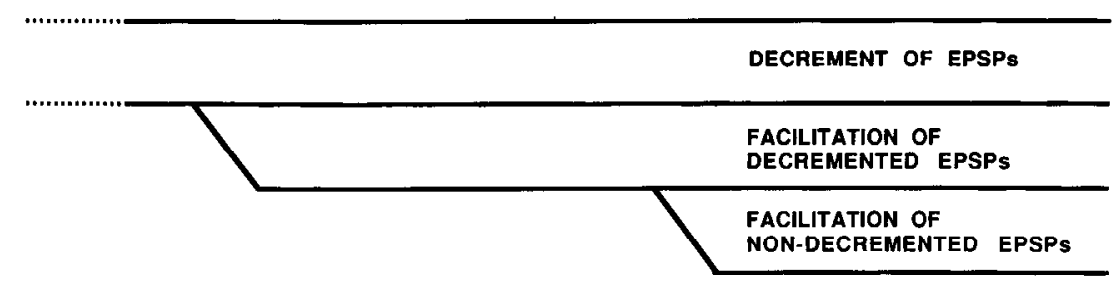

\begin{tabular}{l}
\hline 9 \\
\hline 10
\end{tabular}

DEVELOPMENTAL STAGE

of tail shock emerged in our analysis of nondecremented EPSPs in $\mathrm{R} 2$ prior to the development of sensitization. Specifically, we found that in early stage 12 connective stimulation not only was incapable of facilitating nondecremented synaptic potentials but actually produced significant inhibition of those EPSPs. This observation further strengthens the parallels between the different forms of behavioral plasticity observed during development and the cellular analogs that emerge in close register with them. In their analysis of the emergence of the cellular analog of dishabituation, Rayport and Camardo (1984) made a similar observation of inhibitory effects in R2 in stage 9 juvenile animals. These authors found that when facilitation of decremented EPSPs in R2 was absent in this early developmental stage, connective stimulation produced a short-lived depression of peak EPSP amplitude. Thus, in both stage 9 and early stage 12 , when the facilitatory processes underlying dishabituation and sensitization respectively have not yet emerged, an inhibitory effect of connective stimulation is revealed.

These observations suggest that tail shock (or its physiological analog, connective stimulation), which is normally considered to be a facilitatory stimulus, actually activates facilitatory and inhibitory processes in parallel. This possibility is supported by the recent observation in adult Aplysia that strong tail shock can produce transient depression of the monosynaptic EPSP from siphon sensory neurons and that connective stimulation can produce transient narrowing of the action potential in sensory neurons (Mackey et al., in press). It is possible that a significant component of the inhibitory effects of tail shock we observed involves shunting of the synaptic currents generated in the postsynaptic cell. However, in our experiments the inhibitory effects of connective stimulation could, in some cases, significantly outlast the depolarization produced by the connective stimulation. This suggests that an active inhibitory process produced by connective stimulation may also contribute to the synaptic depression. In adult Aplysia, Hawkins et al. (1981) identified an inhibitory interneuron (L16) in the gill and siphon withdrawal circuit that produces its effects both by inhibiting excitatory interneurons (L22 and L23) and possibly also by mediating recurrent inhibition in the siphon sensory neurons. This inhibitory interneuron sends its axons up one and sometimes both pleuro-abdominal connectives; therefore, it is possible that this cell was activated in our experiments when we stimulated the right connective to activate the tail input to the ganglion. Thus, the interesting possibility emerges that at least part of the inhibitory effects of tail shock we observed may be mediated by the identified inhibitory interneuron $\mathrm{L} 16$, whose effects may developmentally precede the mechanism responsible for sensitization in the reflex.

A candidate transmitter that may contribute to the inhibitory process is the neuropeptide FMRFamide (Price and Greenberg, 1977). This peptide has been shown to produce presynaptic inhibition of transmitter release, as well as a slow hyperpolarization and reduction of action potential duration, in Aplysia sensory neurons (Abrams et al., 1984; Belardetti et al., 1987). Moreover, it has recently been shown that FMFRamide exerts its actions on the sensory neurons by openings a class of $\mathrm{K}^{+}$ channels ( $\mathrm{S}$ channels) that are closed by the facilitatory neurotransmitter 5-HT (Belardetti et al., 1987). Since FMRFamide is implicated in presynaptic inhibition in adult Aplysia sensory neurons, it will be interesting to examine the role of this neuropeptide in modulation of synaptic transmission in juvenile animals, especially in developmental stages when facilitatory processes have not yet emerged. In conjunction with cellular physiological experiments, immunocytochemical approaches, which have already proved instructive in examining the emergence of facilitatory transmitters, such as 5-HT, in juvenile Aplysia (Goldstein et al., 1984; Kistler et al., 1985; Nolen et al., 1986) will be a useful tool to determine the emergence and location of neurons expressing this candidate inhibitory transmitter during development.

\section{Cellular mechanism of presynaptic facilitation in adult} Aplysia involves two components

There is considerable evidence indicating that presynaptic facilitation of transmitter release from sensory neurons in Aplysia contributes significantly to both dishabituation and sensitization 
(for reviews, see Carew, 1987; Hawkins et al., 1987). There is also considerable evidence showing that presynaptic facilitation is produced by broadening of the action potential in the sensory neurons through a reduction of a specific $\mathrm{K}^{+}$current (the $\mathrm{S}$ current) in response to several facilitatory transmitters (Klein and Kandel, 1978, 1980; Klein et al., 1982; Siegelbaum et al., 1982; Abrams et al., 1984; Schuster et al., 1985). However, it has recently become appreciated that, in addition to spike broadening, there is a second component of the cellular mechanism of presynaptic facilitation, a component that is likely to involve $\mathrm{Ca}^{+2}$ handling and transmitter mobilization. For example, Boyle et al. (1984) found that presynaptic facilitation in Aplysia sensory neurons was associated with an increase in $\mathrm{Ca}^{+2}$ transients (as measured with the indicator Arsenazo III) that was independent of changes in $\mathrm{K}^{+}$channels. Moreover, Gingrich and Byrne (1985) concluded that, in addition to spike broadening, a process involving transmitter mobilization also contributes importantly to presynaptic facilitation, especially when the sensory neuron action potential is prolonged. Finally, Hochner and colleagues (1986b), have shown that at normal levels of transmitter release, spike broadening in the sensory neurons accounts for most of the presynaptic facilitation produced by connective stimulation or by 5-HT; however, when the release of transmitter is strongly depressed by prior repeated activation of the sensory neurons, increasing the duration of the action potential (or a depolarizing command potential under voltage clamp) has little effect on transmitter release. Nonetheless, 5-HT or connective stimulation can still enhance the release of transmitter. These observations led Hochner et al. (1986b) to propose that 2 components playing different roles in behavioral plasticity contribute to presynaptic facilitation: The first component, spike broadening, would contribute importantly to facilitation of nondecremented synaptic potentials, and thus to behavioral sensitization; the second component, involving transmitter mobilization, would contribute importantly to facilitation of depressed synaptic potentials, and thus to behavioral dishabituation.

By examining the kinetics of the EPSP produced by the release of transmitter from the sensory neurons, Hochner and colleagues (1986b) were further able to estimate the relative contribution of the 2 components of presynaptic facilitation to the facilitation of decremented and nondecremented EPSPs. Specifically, facilitation involving changes in the time-to-peak of the EPSP could be associated with changes in spike broadening, while facilitation involving changes in the rate-of-rise of the EPSP were taken to reflect changes in transmitter mobilization (Hochner et al., 1986a). Using this analysis, Hochner and colleagues (1986b) found that in facilitation of nondecremented EPSPs (analogous to behavioral sensitization) spike broadening accounted for about $70 \%$ of the facilitation and transmitter mobilization about $30 \%$. The converse was true for facilitation of depressed EPSPs (analogous to behavioral dishabituation): Spike broadcning accounted for about $30 \%$ and transmitter mobilization for about $70 \%$. Thus, the experiments of Hochner and colleagues $(1986 \mathrm{a}, \mathrm{b})$ suggest that the behavioral processes of dishabituation and sensitization share aspects of 2 common mechanisms: spike broadening, which predominates in sensitization, and transmitter mobilization, which predominates during dishabituation. Recently, Gingrich and Byrne (1987) have described a mathematical model that demonstrates that transmitter mobilization in conjunction with spike broadening can account for the physiological results described by Hochner et al. (1986b).

\section{Cellular analysis of the development of presynaptic facilitation}

The experiments described above indicate that in adult Aplysia the 2 components of presynaptic facilitation, although making different relative contributions to the analogs of dishabituation and sensitization, are both present at the same time and contribute in varying amounts, depending upon the level of synaptic depression. Our behavioral and cellular experiments examining the development of dishabituation and sensitization suggest that these 2 processes may emerge independently during development. Thus, it is possible that, at a particular juvenile stage in Aplysia (e.g., early stage 12), one process might be developmentally isolated from the other. The ability to analyze one process in the complete absence of the other may provide important insights into the detailed mechanism of the process that emerges first (perhaps transmitter mobilization) underlying dishabituation, as well as the effects of the subsequent expression of the second process (perhaps spike broadening) underlying sensitization. Important clues concerning the developmental assembly of the 2 components of presynaptic facilitation may come from an analysis of the kinetics of release of transmitter from sensory neurons in different juvenile stages.

Another important question concerns the developmental expression of the cellular and molecular mechanisms that contribute to dishabituation and sensitization. Several candidate mechanisms may account for the development of these 2 processes. For example, the development of particular facilitatory transmitters may play a key role. Although $5-\mathrm{HT}$ is a possible candidate, it is unlikely to differentiate between dishabituation and sensitization since (1) 5-HT can facilitate decremented and nondecremented EPSPs (Brunelli et al., 1976; Hochner et al., 1986a); (2) 5-HT activates both spike broadening and transmitter mobilization in the adult (Hochner et al., 1986b); and (3) 5-HT-containing neurons are present very early in juvenile development (Nolen et al., 1986), at a time when dishabituation first emerges but long before the emergence of sensitization (Rankin and Carew, 1987). Another possible candidate mechanism to differentiate between dishabituation and sensitization would be the development of either cAMP synthetic capability or the ability of specific levels of cAMP to mediate different forms of facilitation. In the adult, the injection of cAMP (Brunelli et al., 1976) or of the cAMP-dependent protein kinase (Castellucci et al., 1982) into the sensory neuron is known to facilitate decremented EPSPs (analogous to dishabituation) and sometimes to produce facilitation of the EPSP to levels above control, suggesting that it can mimic a sensitization-like process. This, in turn, suggests the intriguing possibility that some step after the production of cAMP may emerge late in development and account for the emergence of sensitization. For example, it is possible that the $\mathrm{S}$ channel is not expressed in sensory neurons until late in juvenile development. Finally, in addition to a number of testable hypotheses derived from our current understanding of mechanisms underlying presynaptic facilitation, it is possible that a developmental analysis might reveal novel mechanisms, not predicted by current models, that contribute importantly to the expression of dishabituation and sensitization.

\section{References}

Abrams, T. W., V. F. Castellucci, J. S. Camardo, E. R. Kandel, and P. E. Lloyd (1984) Two endogenous neuropeptides modulate the gill and siphon withdrawal reflex in Aplysia by presynaptic facilitation 
involving cAMP-dependent closure of a serotonin-sensitive potassium channel. Proc. Natl. Acad. Sci. USA 81: 7956-7960.

Belardetti, F., E. R. Kandel, and S. A. Siegelbaum (1987) Neuronal inhibition by the peptide FMRFamide involves opening of $\mathrm{S} \mathrm{K}^{+}$ channels. Nature 325: 153-156.

Boyle, M. B., M. Klein, S. J. Smith, and E. R. Kandel (1984) Serotonin increases intracellular $\mathrm{Ca}^{++}$in voltage-clamped sensory neurons of Aplysia californica. Proc. Nat1. Acad. Sci. USA 81: 7642-7646.

Brunelli, M., V. F. Castellucci, and E. R. Kandel (1976) Synaptic facilitation and behavioral sensitization in Aplysia: Possible role of serotonin and cAMP. Science 194: 1178-1181.

Carew, T. J. (1987) Cellular and molecular advances in the study learning in Aplysia. In The Neural and Molecular Bases of Learning, J. P. Changeaux and M. Konishi, eds., pp. 177-204, Wiley, New York.

Carew, T. J., V. F. Castellucci, and E. R. Kandel (1971) An analysis of dishabituation and sensitization of the gill-withdrawal reflex in Aplysia. Int. J. Neurosci. 2: 79-98.

Carew, T. J., V. F. Castellucci, and E. R. Kandel (1979) Sensitization in Aplysia: Restoration of transmission in synapses inactivated by long-term habituation. Science 205: 417-419.

Castellucci, V. F., I. Kupfermann, H. Pinsker, and E. R. Kandel (1970) Neuronal mechanisms of habituation of the gill withdrawal reflex in Aplysia. Science 67: 1445-1448.

Castellucci, V. F., T. J. Carew, and E. R. Kandel (1978) Cellular analysis of long-term habituation of the gill-withdrawal reflex of $\mathrm{Aply}$ sia californica. Science 202: 1306-1308.

Castellucci, V. F., A. Nairn, P. Greengard, J. H. Schwartz, and E. R. Kandel (1982) Inhibitor of adenosine 3':5'-monophosphate-dependent protein kinase blocks presynaptic facilitation in Aplysia. J. Neurosci. 2: 1673-1681.

Gingrich, K. J., and J. H. Byrne (1985) Simulation of synaptic depression, posttetanic potentiation, and presynaptic facilitation of synaptic potentials from sensory neurons mediating gill-withdrawal reflex in Aplysia. J. Neurophysiol. 53: 652-669.

Gingrich, K. J., and J. H. Byrne (1987) Mathematical model of two cellular mechanisms contributing to dishabituation and sensitization in Aplysia. Soc. Neurosci. Abstr. 13 (in press).

Goldstein, R., H. B. Kistler, Ir., H. W. M. Steinbusch, and J. H. Schwartz (1984) Distribution of serotonin-immunoreactivity in juvenile $A p l y$ sia. Neuroscience 11: 535-547.

Hawkins, R. D., V. F. Castellucci, and E. R. Kandel (1981) Interneurons involved in mediation and modulation of gill-withdrawal reflex in Aplysia. I. Identification and characterization. J. Neurophysiol. 45: 304-314.

Hawkins, R. D., G. A. Clark, and E. R. Kandel (1987) Cell biological studies of learning in simple vertebrate and vertebrate systems. In Handbook of Physiology, Section I: The Nervous System, Vol. 6, F. Plum, ed., American Physiological Society, Bethesda, MD.

Hochner, B., M. Klein, S. Schacher, and E. R. Kandel (1986a) Actionpotential duration and the modulation of transmitter release from the sensory neurons of Aplysia in presynaptic facilitation and behavioral sensitization. Proc. Natl. Acad. Sci. USA 83: 8410-8414.

Hochner, B., M. Klein, S. Schacher, and E. R. Kandel (1986b) Additional component in the cellular mechanism of presynaptic facilitation contributes to behavioral dishabituation in Aplysia. Proc. Natl. Acad. Sci. USA 83: 8794-8798.

Kandel, E. R. (1976) The Cellular Basis of Behavior, Freeman, San Francisco.

Kandel, E. R., and L. Tauc (1965) Heterosynaptic facilitation in neurones of the abdominal ganglion of Aplysia depilans. J. Physiol. (Lond.) 181: $1-27$.
Kistler, H. B., Jr., R. D. Hawkins, J. Koester, H. W. M. Steinbusch, E. R. Kandel, and J. H. Schwartz (1985) Distribution of serotoninimmunoreactive cell bodies and processes in the abdominal ganglion of mature Aplysia. J. Neurosci. 5: 72-80.

Klein, M., and E. R. Kandel (1978) Presynaptic modulation of voltagedependent $\mathrm{Ca}^{++}$current: Mechanism for behavioral sensitization. Proc. Natl. Acad. Sci. USA 75: 3512-3516.

Klein, M., and E. R. Kandel (1980) Mechanism of calcium current modulation underlying presynaptic facilitation and behavioral sensitization in Aplysia. Proc. Natl. Acad. Sci. USA 77: 6912-6916.

Klein, M., J. Camardo, and E. R. Kandel (1982) Serotonin modulates a specific potassium current in the sensory neurons that show presynaptic facilitation in Aplysia. Proc. Natl. Acad. Sci. USA 79: 57135717.

Kriegstein, A. R. (1977a) Development of the nervous system of Aplysia californica. Proc. NatI. Acad. Sci. USA 74: 375-378.

Kriegstein, A. R. (1977b) Stages in the post-hatching development of Aplysia californica. J. Exp. Zool. 199: 275-288.

Kupfermann, I., H. Pinsker, V. F. Castellucci, and E. R. Kandel (1970) Neuronal correlates of habituation and dishabituation of the gillwithdrawal reflex in Aplysia. Science 167: 1743-1745.

Mackey, S. L., D. L. Glanzman, S. A. Small, A. M. Dyke, E. R. Kandel, and R. D. Hawkins (1987) Aversive stimuli produce inhibition as well as sensitization of the siphon withdrawal reflex of Aplysia: A possible behavioral role for presynaptic inhibition mediated by the peptide FMRF amide. Proc. Natl. Acad. Sci. USA (in press).

Nolen, T. G., J. A. Mindell, and T. J. Carew (1986) Development of neurotransmitters implicated in learning in Aplysia. Soc. Neurosci. Abstr. 12: 399.

Nolen, T. G., E. A. Marcus, and T. J. Carcw (1987) Devclopment of learning and memory in Aplysia. III. Central neuronal correlates. J. Neurosci. 7: 144-153.

Price, D. A., and M. J. Greenberg (1977) Structure of a molluscan cardioexcitatory neuropeptide. Science 197: 670-671.

Rankin, C. H., and T. J. Carew (1987) Development of learning and memory in Aplysia. II. Habituation and dishabituation. J. Neurosci. 7: 133-143.

Rankin, C. H., and T. J. Carew (1988) Dishabituation and sensitization emerge as separate processes during development in Aplysia. J. Neurosci. 8: 197-211.

Rankin, C. H., M. Stopfer, E. A. Marcus, and T. J. Carcw (1987) Development of learning and memory in Aplysia. I. Functional assembly of gill and siphon withdrawal. J. Neurosci. 7: 120-132.

Rayport, S. G. (1981) Development of the functional and plastic capabilities of neurons mediating a defensive behavior in Aplysia. Ph.D. Thesis, Columbia University, New York, NY.

Rayport, S. G., and J. S. Camardo (1984) Differential emergence of cellular mechanisms mediating habituation and sensitization in the developing Aplysia nervous system. J. Neurosci. 4: 2528-2532.

Schuster, M. J., J. S. Camardo, S. A. Siegelbaum, and E. R. Kandel (1985) Cyclic AMP-dependent protein kinase closes the serotoninsensitive $\mathrm{K}^{+}$channels of Aplysia sensory neurons in cell-free membrane patches. Nature 313: 392-395.

Siegel, S. (1956) Nonparametric Statistics for the Behavioral Sciences, McGraw-Hill, New York.

Siegelbaum, S. A., J. S. Camardo, and E. R. Kandel (1982) Serotonin and cyclic AMP close single $\mathrm{K}^{+}$channels in Aplysia sensory neurons. Nature 299: 413-417.

Sokal, R. R., and F. J. Rohlf (1969) Biometry, Freeman, San Francisco.

Stopfer, M., and T. J. Carew (1988) Development of sensitization in the escape locomotion system of Aplysia. J. Neurosci. 8: 223-230. 